\title{
Capital social comunitario: recurso promotor en los emprendimientos agroecológicos
}

Community Social Capital: Promoter Resource in Agro-Ecological Ventures

Capital social comunitário: recurso promotor em empreendimentos agroecológicos

\author{
Marco Polo Cevallos Suarez \\ Universidad Andina Simón Bolivar, Sede Ecuador \\ cevallos1963@hotmail.com \\ José Gregorio Mendoza Mendoza \\ Universidad de Los Andes, sede Trujillo (Venezuela) \\ jgmendoz@ula.ve
}

DOI: https://doi.org/10.32719/25506641.2019.5.4

Fecha presentación: 24 de enero de 2019 • Fecha de aceptación: 29 de marzo de 2019

Artículo de investigación

Licencia Creative Commons

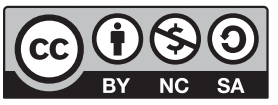




\section{Resumen}

El objetivo es destacar los aportes del capital social comunitario a emprendimientos agroecológicos. Se desarrolló un procedimiento metodológico que incluyó una revisión documental bajo una episteme racionalista deductiva. Los recursos asociados al capital social comunitario-campesino conducen, promueven y movilizan procesos de transformación social en los sistemas de producción agroecológicos. De los resultados se concluye que la cooperación -confianza y asociatividad en organizaciones campesinas agroproductivasconstituye una condición necesaria para hacer viable la gestión de los emprendimientos agroecológicos y una precondición que facilita su inicio.

Palabras clave: cooperación, asociatividad, transformación social, organización campesina. JEL: Z13 Normas sociales y capital social; redes sociales; O13 Agricultura, recursos naturales.

\section{Abstract}

The objective is to highlight the contributions of community social capital to agro-ecological ventures. A methodological procedure that included a documentary review under a deductive rationalist episteme was developed. The resources associated with community-peasant social capital lead, promote and mobilize social transformation processes in agro-ecological production systems. From the results it is concluded that cooperation, trust and associativity in agro-productive peasant organizations, constitutes a necessary condition to make the management of agro-ecological ventures viable and a precondition that facilitates their initiation.

Keywords: cooperation, associativity, social transformation, peasant organization.

JEL: Z13 Social norms and social capital; social networks; O13 Agriculture, natural resources.

\section{Resumo}

O objetivo é destacar as contribuições do capital social da comunidade para os empreendimentos agroecológicos. Um procedimento metodológico foi desenvolvido e incluiu uma revisão documental sob uma episteme racionalista dedutiva. Os recursos associados ao capital social camponês-comunitário levam, promovem e mobilizam processos de transformação social em sistemas de produção agroecológicos. A partir dos resultados conclui-se que a cooperação-confiança e associatividade em organizações camponesas agro-produtivas-constitui uma condição necessária para viabilizar o manejo de empreendimentos agroecológicos e uma precondição que facilite sua iniciação.

Palavras-chave: cooperação, associatividade, transformação social, organização camponesa. JEL: Z13 Normas sociais e capital social; redes sociais; O13 Agricultura, recursos naturais. 


\section{Introducción}

П $\mathrm{s}$ imposible negar que desde los albores de la humanidad existiera una relación simbiótica entre el hombre y la naturaleza, evidente en diversas latitudes y culturas; sin embargo, en la actualidad es también evidente un divorcio en esa relación que ha traído como consecuencia el creciente deterioro ambiental que hoy padece el mundo. Cada vez más se están reconociendo los estrechos vínculos existentes entre ecosistemas y sistemas sociales. De hecho, en un proceso de coevolución los sistemas humanos y los ecosistemas se han ido moldeando y adaptando conjuntamente, convirtiéndose en un sistema integrado de humanos en la naturaleza denominado sistema socio-ecológico (SSE); este marco teórico fue planteado por Berkes y Folke (1998), con el objetivo de dar el mismo peso tanto a la dimensión social como a la natural y desde este enfoque se visualizan los sistemas de producción agroecológicos (Altieri y Nicholls 2007).

En las últimas décadas, en Latinoamérica, y específicamente en Ecuador, se han impulsado procesos de transición de sistemas agrícolas de producción convencional a sistemas de producción agroecológicos, con el propósito de fomentar la soberanía alimentaria en concordancia con el cuidado del ambiente. Esto bajo el patrocinio de ONG y, en los últimos años, desde el gobierno nacional con la promulgación de la Ley de Soberanía Alimentaria del año 2009.

Tales procesos están dirigidos a comunidades rurales, organizaciones campesinas y pequeños productores por medio de mecanismos tales como sello de agricultura familiar campesina, programas de capacitación, recuperación de la agrobiodiversidad, manejo de huertos familiares, granjas integrales, uso de tecnologías en control integrado de plagas y enfermedades, recuperación de semillas autóctonas, manejo ecológico de suelos, implantación de sistemas de regadío a nivel parcelario, manejo de cosecha y valor 
agregado a la producción, entre otros. Sin embargo, no ha sido posible observar un desarrollo de tales sistemas de producción como se esperaba. De hecho, según estudios de Heifer-Ecuador (2014), se estima que en Imbabura existen 673 productores agroecológicos que representan apenas el 1,9\% del total de unidades de producción agropecuaria de la provincia.

Una revisión exploratoria de las causas indica que, hasta el momento, las acciones han sido enfocadas, principalmente, a procesos tecnológicos pero con bajo grado de atención a importantes problemas sociales de las organizaciones campesinas de producción agroecológica, tales como la reducida cooperación social, el bajo grado de asociatividad y confianza en las organizaciones campesinas, y el inadecuado manejo de los recursos naturales en las unidades de producción, así como el poco reconocimiento y atención que otorgan las instituciones gubernamentales al gran aporte que brinda estos sistemas de producción, en la obtención de alimentos sanos, cuidado del ambiente y mejora en la salud de las familias campesinas y consumidores.

En este contexto se hace necesario indagar, por una parte, la visión que tienen algunos pensadores, relacionada con la importancia y validez de los recursos asociados al capital social (cooperación, asociatividad y confianza) en las acciones colectivas de las organizaciones campesinas, tomando en cuenta que tales recursos son elementos fundamentales para alcanzar una soberanía alimentaria sana a partir de la construcción de estilos alimentarios sustentables. Y, por la otra, conocer los niveles de referencia que tienen diversos especialistas sobre la trasformación social que implica articular y promover relaciones entre las instituciones y las organizaciones campesinas para el fortalecimiento del capital social en procesos de emprendimientos y transición agroecológicos.

La transición agroecológica es un proceso de cambio en las prácticas agrícolas y la readecuación biológica de un sistema agropecuario, tendiente a la recuperación de los principios agroecológicos para lograr resultados equilibrados en torno a la producción, la independencia de insumos externos, especialmente agroquímicos y la restauración de todos los procesos ecológicos y sociales que le permitan acercarse a la sustentabilidad (CET 2018). Desde una perspectiva social, Caporal y Costabeber (2004) señalan que la transición agroecológica es también un proceso social que implica no solo la búsqueda de una mayor racionalización económico-productiva, sino también 
un cambio en las actitudes y valores de los actores sociales en relación al manejo y conservación de los recursos naturales.

Calle, Vara y Cuéllar (2006) indican que existen elementos importantes en un proceso de transición social agroecológica como la cooperación, la participación y los bienes comunes. Según estos autores, la cooperación se refiere a la confianza y reciprocidad; la confianza puede ser entendida como una actitud que permite la cesión voluntaria del control de recursos (Durston 2002) y la cooperación como una acción colectiva orientada al logro de objetivos comunes (Miranda y Monzó 2003).

Desde la perspectiva del capital social, Putnam (1993) afirma que las acciones colectivas surgen como resultado de las relaciones sociales, de la confianza y la cooperación entre los individuos, y son la base para aunar esfuerzos que beneficien a toda la comunidad. Las organizaciones, en su conformación, persiguen propósitos y, para alcanzarlos, requieren hacer uso de los recursos que dispone el capital social. Por otro lado, Gómez, Vera y Garrido (2012) señalan que las relaciones sociales pueden afectar la sostenibilidad económica de los agricultores al influir, a través, por ejemplo, de la información obtenida por medio de esas redes de contacto, en las prácticas agrícolas y en su propensión a adoptar nuevas tecnologías. Los agricultores pueden, de esa manera, aprender nuevas técnicas y conocimientos, obtener una formación informal de otros que ya han adoptado determinada medida, e incluso lograr asistencia oficial para poner en marcha distintas prácticas.

En virtud de ello, el trabajo que se presenta asume como principal objetivo la perspectiva analítica de los referentes teórico-metodológicos que sustentan la validez y características del capital social comunitario que promueven emprendimientos agroecológicos en las organizaciones campesinas, para generar una perspectiva de análisis que visualice la importancia y necesidad de incluir la dimensión social en el contexto del desarrollo de sistemas de producción agroecológicos.

En la exploración bibliográfica que fundamenta la investigación, se inicia con una definición del capital social y una exposición de tendencias y visiones que giran alrededor de los desafíos que entraña reconocer la validez de este recurso, se indaga los aportes conceptuales y controversias del capital social como stock propio de la sociedad y sus diversas formas y manifestaciones en las relaciones sociales. Se analiza la capacidad efectiva de movi- 
lizar productivamente y en beneficio los activos de este capital para lograr transformaciones fructíferas. Posteriormente se muestra un compendio de los aportes teóricos y metodológicos del capital social comunitario-campesino con los que se puede promover el desarrollo de los sistemas de producción agroecológicos.

Se analiza la agroecología como enfoque holístico y estudio ecosistémico, se exponen las razones que permiten reorientar la agricultura convencional hacia un modelo de producción socialmente justo y sostenible desde una visión ambiental, que considera la cultura como factor relevante. Más adelante, se indaga la transformación social agroecológica, lo que conduce a que en la revisión bibliográfica se busque el vínculo con los recursos del capital social para trascender a los emprendimientos agrícolas de distinción social, acepción que implica confianza, solidaridad y cooperación. Finalmente se presentan las conclusiones y futuras investigaciones.

\section{Metodología}

Para el presente trabajo se utilizó una investigación no experimental transversal descriptiva; metodológicamente, requirió el análisis de enfoques y teorías que permitieron identificar, bajo una episteme racionalista deductivo, los recursos del capital social, la relación y articulación con procesos de transformación productiva agroecológica.

\section{Surgimiento y relevancia del capital social}

Desde la década de 1980 han surgido una cantidad de pensadores que han sentado las bases para la discusión y el estudio de capital social. En sus escritos se reconoce la importancia y validez de este capital intangible en la perspectiva del desarrollo y su adecuación al marco de análisis de la teoría de las decisiones racionales. Es así como Bourdieu (1985) lo define como el agregado de los recursos reales o potenciales ligados a la posesión de una 
red durable de relaciones más o menos institucionalizadas de reconocimiento mutuo.

Por su parte, Coleman (1990) asevera que el capital social responde a un conjunto de entidades formadas por redes cuya misión es la de conducir hacia una acción a las personas o a una colectividad bajo una estructura social definida (vertical u horizontal), clasificándolo en capital social individual y colectivo. El primero está definido por el grado de integración, redes sociales, relaciones sociales, expectativas de reciprocidad y confianza entre los individuos; mientras que el segundo está caracterizado por la confianza, la solidaridad, la asociatividad y el beneficio común. Los dos autores resaltan e incorporan en sus conceptos el término red como un mecanismo de conexión e interrelación que se construye paulatinamente entre las personas y de forma colectiva.

Más adelante, Putnam (1993) añade al concepto el hecho de que las redes de compromiso cívico y los niveles de asociatividad, sumados a los niveles de confianza y normas de reciprocidad, permiten crear y mantener la riqueza y fortaleza de un tejido social dentro de una organización, además señala que dicho capital debe ir de la mano en la búsqueda del desarrollo. No obstante, Newton (1997) considera que el capital social puede ser visto como un fenómeno subjetivo, compuesto de valores y actitudes que influyen en la manera cómo las personas se relacionan entre sí, incluyendo la confianza y las normas de reciprocidad, así como las actitudes y valores que ayudan a las personas trascender relaciones conflictivas y competitivas para conformar relaciones de cooperación y ayuda mutua. Estos autores coinciden en que la confianza y las normas de reciprocidad son actitudes y valores que contribuyen a fortalecer las relaciones sociales y a mitigar conflictos en las organizaciones. Es a partir de los postulados de Putnam que la expresión capital social cobra una mayor importancia y cada vez académicos de distintas disciplinas, así como organismos internacionales como el Banco Mundial, CEPAL, BID y Naciones Unidas, lo incorporan en sus estudios.

Miranda y Monzó (2003) explican que las definiciones de capital social pueden distinguirse entre aquellas que se centran en aspectos culturales-valóricos y aquellas que lo sitúan en las estructuras sociales; es decir, en el ámbito conductual, relacional u organizacional, resaltando sus manifestaciones en diferentes niveles de agregación social (micro, meso y macro). 
El capital social alude, también, a la forma de inserción de los individuos y comunidades en estructuras interrelacionadas, y a la forma en que esta inserción deriva en oportunidades individuales y colectivas (Parker 2001). Bajo la visión de Kliksberg (2002), el capital social busca alcanzar la capacidad de una sociedad para producir concertaciones sociales amplias a su interior a los fines de generar redes articuladoras de los esfuerzos de sus diversos sectores; las fuerzas existentes de una sociedad para impulsar el trabajo voluntario en iniciativas de utilidad general; su cultura, y la presencia de valores éticos orientados hacia la solidaridad, la construcción positiva, la cooperación y la equidad. Por su parte, Arriagada (2003) lo asume como la capacidad efectiva de movilizar, productivamente y en beneficio del conjunto, los recursos asociativos que radican en las distintas redes sociales a las que tienen acceso los miembros del grupo. Señala además que el capital social comprende varios factores, tales como el clima de confianza social, el grado de asociatividad, la conciencia cívica, los valores éticos y la cultura, entendida como la manera de vivir juntos.

El planteamiento de Arriagada (2003) y Parker (2001) es coherente con la visión de Kliksberg (2002), al señalar que el capital social busca producir concertaciones sociales y constituye un activo en el cual todas las personas, grupos y organizaciones pueden obtener un máximo beneficio, pues, al ser un conjunto de recursos reales o potenciales, aportan dinámicamente, en forma individual o colectiva, al desarrollo de las organizaciones sociales.

Sin embargo, si bien las relaciones crean confianza y solidaridad, no todas las relaciones construyen capital social. Las relaciones de simple intercambio o encuentro entre individuos que no continúan con esa relación no construyen capital social (Pizzorno 2003). La riqueza, en términos de capital social, está dada por el número de relaciones que posee un individuo, una comunidad, o una sociedad y por el nivel de dependencia entre unos y otros (Piselli 2003). Esto refleja que el capital social constituye un stock que posee la sociedad en sus diversas formas y manifestaciones y que pasa muchas veces de manera desapercibida y requiere estar en constante construcción para percibir sus beneficios.

No obstante, Putzel (1997) y Portes (1998) ya afirmaban que primero es necesario resolver los dilemas conceptuales implícitos en los discursos de capital social. El primero señala una peligrosa tautología en este discurso ya 
que trata de explicar el capital social como causa y efecto, pretendiendo que su existencia se infiere a partir de los efectos positivos previamente postulados. El segundo considera que existe un lado oscuro (dark side) del capital social, al manifestar que, si bien su estímulo tiene consecuencias positivas como ser fuente de control social, de apoyo familiar y de beneficios basados en redes sociales no familiares, también tiene consecuencias negativas, tales como la exclusión de aquellos que no forman parte de las estructuras sociales, las restricciones a la libertad individual (por ejemplo: en algunos grupos existe un alto control social restrictivo), las normas niveladoras hacia abajo para pertenecer a un grupo implica seguir acciones que no permiten el progreso individual y las excesivas exigencias de la comunidad al individuo que impiden la acumulación de capital en los miembros exitosos. Estos autores resaltan que es necesario distinguir los efectos negativos o consecuencias no deseables que el capital social puede ocasionar, como organizaciones criminales o fenómenos excluyentes.

Por su parte, Putnam (en Ramírez 2005) categoriza el capital social en: a) formal; b) informal; c) denso; d) tenue; e) vuelto hacia adentro; f) vuelto hacia afuera; g) vinculante; y h) que no tiene puentes. Por otro lado, Arriagada (2003), de acuerdo a los ejes principales del capital social, tomando como criterio la combinación de la naturaleza del liderazgo existente en una comunidad con la naturaleza de las redes internas que posee y externas a que tiene acceso, plantea la siguiente clasificación: a) capital social restringido: en un grupo que tiene redes sociales internas y cuenta con un liderazgo para su manejo; b) capital social en desarrollo: cuando en un grupo, en adición a lo anterior, hay un liderazgo para abordar el mundo externo, pero no se forma parte de las redes existentes o aun cuando se forma parte de las redes externas, se carece de un liderazgo para captar los posibles beneficios; y c) capital social ampliado: cuando articula armónicamente redes internas y externas y cuenta con un liderazgo capaz de movilizar los recursos existentes en ambos tipos de redes.

Como se puede evidenciar, la densidad de investigaciones sobre el capital social y sus relaciones con el desarrollo es variada. Buena parte de esta labor ha tenido un carácter conceptual, es decir, ha tratado de comprender qué es el capital social, cómo funciona y cómo se origina. Este bagaje de información, según López, Martin y Romero (2007), ha sido procesado de 
distintas maneras dependiendo del objeto del estudio de cada proyecto de investigación, de las variables que definen el término, el nivel de análisis de los trabajos micro, meso y macro, de los beneficiarios del capital social individual o colectivo y de los fundamentos teóricos que sustentan los diferentes proyectos. Por lo tanto, en esta panorámica dispersa de reflexiones, enfoques y falta de consensos de los investigadores alrededor de la conceptualización del capital social se puede decir que el mismo aún está en construcción.

Mientras dure el debate epistemológico y metodológico, dada la complejidad del tema, el capital social continúa dando muestras de su presencia y acción efectiva positiva; existe un denominador común en medio de esta trama: el capital social, a diferencia de los otros capitales, aumenta con su utilización, cuanto más se emplea, más se incrementa y se fortalece. A manera de indicador, el índice de Capital Social (ICS) impulsado por el Programa de las Naciones Unidas para el Desarrollo puede servir de referente teórico y metodológico para evaluar y comparar la confianza social, asociatividad y reciprocidad (PNUD 2000).

\section{El capital social comunitario-campesino}

Esta forma de capital social más específica surge de una revisión hecha por Durston (2000) a los argumentos de Putnam (1993). El autor sostiene que esta forma de capital no es un recurso individual, sino una forma de institucionalidad social del conjunto, en este caso, de la comunidad local. Aquí, los participantes tienen como objetivo, en forma explícita o implícita, el bien común, aunque no necesariamente lo alcancen. El autor señala que existen dos visiones de la existencia de capital social en el campo. La una tiene un enfoque romántico de la comunidad rural solidaria y cooperativa; $y$, la contraria, postula que estas prácticas solidarias fueron ya destruidas por la represión e implantación de las políticas neoliberales, que privilegian el mercado y tienen un efecto atomizador sobre las relaciones humanas. En el contexto de la primera visión, Rivas (2007) aclara que el capital social, en lo que queda de campesinado alrededor del mundo, ha existido desde hace muchísimo tiempo y que se ha manifestado históricamente a través de agrupamientos sociales que se resisten a desaparecer como clase y que son los 
mismos que sobreviven favorecidos por los rasgos de relaciones solidarias remanecientes que aún se encuentran en ellos.

En una reinterpretación de Putnam (1993), las normas de reciprocidad y confianza social serían los elementos hipotéticos facilitadores de la coordinación y cooperación para el beneficio mutuo. Sin embargo, Dirven (2003) señala que esta hipótesis no siempre es verdadera; de acuerdo a estudios de varias experiencias en organizaciones campesinas de América Latina, se evidencia que el individualismo, los conflictos, las relaciones de poder y de clientelismo prevalecen o son suficientemente frecuentes para no ignorarlas.

Como se puede apreciar, el capital social comunitario en espacios campesinos ha representado la oportunidad para crear consecuentes análisis y discusiones en torno a la manera en que se manifiesta o expresa el mismo en este tipo de entorno, toda vez que los investigadores han observado que, para unos casos, prevalece el bien común sustentado en la solidaridad y el cooperativismo y, para otros, ha dominado el individualismo y los intereses particulares que han aminorado las prácticas solidarias.

El capital social en el mundo rural, según Martínez (2003), es definido desde dos dimensiones: relacional y procesual. La primera dimensión indica que las prácticas de reciprocidad o cooperación, así como las redes y normas que facilitan una acción colectiva - es decir, las relaciones sociales que se generan entre los productores rurales-, se concretizan entre grupos, e individuos diversos y diferentes. La segunda indica que se trata de un proceso que puede implicar crecimiento o decrecimiento; agregando que no basta con tener capital social, sino, ante todo, de cómo se puede potenciar este recurso en un entorno como el medio rural donde los otros capitales como el económico y humano son escasos.

El planteamiento de Martínez (2003) se armoniza con lo que señala Araque (2013), a través de una investigación realizada en Ecuador con pequeñas y medianas empresas, al expresar que para que se logre un efectivo fortalecimiento empresarial mediante la práctica asociativa se requiere del accionar de un conjunto, tanto de factores económicos como extraeconómicos: confianza, cooperación y valores éticos, entre otros. En tal sentido, las organizaciones en sus diversas modalidades y formalidades dependen no solo de la tecnología y recursos locales disponibles, sino también de numerosos aspectos del sistema social, económico e institucional circundante. 
En cuanto a las funciones del capital social comunitario, Durston (2000) las sintetiza en: a) control social a través de la imposición de normas compartidas por el grupo y el sancionar por oprobio o castigo de individuos transgresores; b) creación de confianza entre los miembros de un grupo; c) cooperación coordinada en tareas que exceden las capacidades de una red; d) resolución de conflictos por líderes o por una judicatura institucionalizada; e) movilización y gestión de recursos comunitarios; f) legitimación de líderes y ejecutivos con funciones de gestión y administración; y g) generación de ámbitos y estructuras de trabajo en equipo.

Por su parte, Flora y Butler (2003), en un análisis del desarrollo comunitario en los Andes, plantean una tipología de capital social sobre la base de la relación horizontal y vertical de las comunidades a nivel interno (intragrupo) y con otras externas (intergrupos) en el ámbito local. Esos tipos son: a) horizontalidad: en donde el aprendizaje lateral es esencial para las redes, pues una comunidad aprende mejor cuando aprende de otra; b) verticalidad: cuando las comunidades están vinculadas a organizaciones y recursos regionales, provinciales y nacionales; c) flexibilidad: cuando el formar parte de una red no es un compromiso definitivo, pues la gente está dispuesta a participar en la medida que pueda lograr algún cambio; y d) permeabilidad de los límites: al ampliarse la comunidad de intereses la comunidad territorial crece a medida que se forman asociaciones y mecanismos de colaboración.

Lo expuesto por Flora y Butler (2003) concuerda con Durston (2002), quien sostiene que el tema clave del capital social comunitario es el grado de asociatividad intercomunitario que dé paso a la participación institucional pública y el empoderamiento del sistema interno de su comunidad, siempre en vistas del desarrollo local. Por lo tanto, la organización no solo ha de ser vista desde una óptica netamente económica, sino, más bien, desde el contexto dentro del cual los habitantes de las comunidades han establecido relaciones de hermandad, solidaridad y cooperación que garantizan, por un lado, la estabilidad organizativa y, por el otro, la eficiencia de la acción colectiva, se refiere a formas de asociatividad que están abiertas a la participación y cooperación de actores del entorno (Razzeto 2007) que se dinamizan desde lo interno para alcanzar objetivos que redundarán en un bien común. Estas asociaciones pueden estar organizadas en diversas modalidades como: comunidades, cooperativas, redes, entre otras. 
Por otro lado, Schejtman y Berdegué (2004) manifiestan que las estrategias de acción colectiva en el sector rural, por lo general, apuntan a objetivos como el mejoramiento de las condiciones materiales de vida; la modificación de las relaciones de poder dentro de los grupos, comunidades u organizaciones rurales; la profundización de la democracia y la expansión de la ciudadanía. En todos los casos, señalan los autores, se trata de propósitos complejos, cuya consecución demanda la existencia o el desarrollo de capacidades sustentadas en el capital social.

Se puede observar que, a diferencia de otros tipos de capital, el capital social comunitario se coloca en la estructura de las relaciones sociales. Se trata, por ello, de una noción que intenta captar una práctica relacional, ya sea en las redes establecidas al interior de la familia, entre la familia, la comunidad o en su entorno comunicacional. Además, este capital ha existido desde siempre y se ha expresado en diversas formas de organización social que, buscando el bien común en función de la reciprocidad, la cooperación y la confianza, se resisten a desaparecer y sobreviven favorecidos por los rasgos de relaciones solidarias que aún se encuentran en ellos.

\section{Agroecología: otra forma de hacer agricultura}

La agricultura y su estudio constituyen una actividad compleja debido a su dependencia de los fenómenos naturales y a la variada gama de conocimientos, teóricos y prácticos, necesarios para el suceso de su emprendimiento (Rivas 2007). De esta manera, en el caso de la agroecología, al juntar el factor ecológico a su ya intrincado campo de estudio y aplicación, al tiempo que se enriquece, también adquiere una mayor complejidad que desafía continuamente los límites de su comprensión.

A partir de esta premisa, se ha observado en las últimas décadas que el sistema socioeconómico y su modelo, basado en la relación producción-consumo, se ha globalizado de forma muy significativa, impactando el sistema biogeofísico del planeta de tal manera que ya se habla de una nueva era geológica llamada Antropoceno (Crutzen 2002). De allí, la necesidad de analizar las interrelaciones complejas que se establecen entre los ecosistemas y las culturas o estudio de las ciencias ambientales. 
En este sentido, el estudio ambiental se basa en dos ejes interrelacionados: el de la ecología y el de la cultura (Ángel 1996; Carrizosa 2001). El primer eje con base teórica sobre el funcionamiento de los ecosistemas, entendidos como tramas complejas de intercambios de materia y flujos de energía e información regulada, tanto por la influencia de leyes termodinámicas como por leyes ecosistémicas de equilibrio dinámico espacial y temporal. El segundo, es decir, la cultura, ampliamente debatida como concepto unificador en las ciencias sociales, explica los procesos adaptativos de los seres humanos a los límites impuestos por los ecosistemas y estudia las causas y efectos de esa intervención humana sobre los ecosistemas (Farhad 2012).

De esta misma manera, es importante la incorporación de nuevos instrumentos de análisis que estén basados en una prospección holística para lograr una interacción eficaz y efectiva entre ciencias sociales y naturales. Entre ellos, el marco de los sistemas socio-ecológicos (SSE), utilizado por primera vez por Berkes y Folke (1998), con el objetivo de dar el mismo peso tanto a la dimensión social como a la natural.

Se denominan SSE aquellos sistemas en los que múltiples componentes culturales, políticos, sociales, económicos, ecológicos, tecnológicos y otros están interactuando. Tal es el caso de los sistemas de producción agroecológicos, caracterizados por ser emprendimientos productivos arraigados en la racionalidad ecológica campesina de la agricultura tradicional, con tecnologías y prácticas de manejo de los recursos naturales relacionadas a su cosmovisión cultural (vínculo que se establece entre el ser humano y la naturaleza), con propias formas de organización para el trabajo y comercialización, basados en dinámicas sociales de cooperación y asociatividad.

A decir de Altieri (1995) y Gliessman (2002), los sistemas de producción fundados en principios agroecológicos son biodiversos, resilientes, eficientes energéticamente, socialmente justos y constituyen la base de una estrategia energética y productiva fuertemente vinculada a la soberanía alimentaria. Los principios básicos para una acertada gestión en los emprendimientos agroecológicos incluyen el reciclaje de nutrientes y energía, la sustitución de insumos externos, el mejoramiento de la materia orgánica y la actividad biológica del suelo, la diversificación de las especies de plantas y los recursos genéticos de los agroecosistemas en tiempo y espacio, la integración de los cultivos con la ganadería, y la optimización de las interacciones y la 
productividad del sistema agrícola en su totalidad, en lugar de los rendimientos aislados de las distintas especies (Gliessman 2002). Esta definición de los sistemas de producción agroecológicos establece que sus principios son universales; en cambio, las tecnologías y prácticas son locales, pues estas difieren de acuerdo a los contextos biofísicos (clima, suelo, biodiversidad) y culturales (costumbres, tradiciones) del territorio.

La agroecología tiene sus bases en la ciencia agrícola, ciencia agraria, en el análisis de agroecosistemas, en estudios sobre el desarrollo rural, en la sociología y antropología y han influido en su concepción y desarrollo, la etnología, los estudios campesinos, el ambientalismo, la economía ecológica y la ecología política (Altieri 1995; Guzmán, González y Sevilla 2000). En palabras de Yurjevic (1996), la agroecología permite mejorar el stock de los recursos naturales, aumentar la productividad de los recursos cultivados y potenciar el stock de recursos humanos, dotando al campesino del conocimiento para ser un productor eficiente. En tal sentido, la agroecología es considerada como ciencia, práctica y movimiento social conducente no solo a una transformación productiva amigable con el ambiente, sino a una trasformación social comunitaria, articulada a los recursos del capital social, tal como se ilustra en el gráfico 1.

Los emprendimientos agroecológicos son impulsados por organizaciones campesinas, con apoyo de ONG y entidades gubernamentales; así, por ejemplo, en Ecuador pequeños productores con emprendimientos agroecológicos se han organizado para incursionar en la venta directa en los mercados locales, principalmente a través de la modalidad de ferias solidarias. La primera experiencia en el país fue en la provincia de Pichincha, donde desde el año 2000 se inició la comercialización de productos en tiendas y puntos de venta en espacios públicos y en el año 2002 se iniciaron las ferias agroecológicas en otras zonas del país (Coordinadora Ecuatoriana de Agroecología 2010).

\section{Agroecología y capital social campesino}

La Coordinadora Europea Vía Campesina (2014) percibe la transformación social desde la producción y lucha campesina que va más allá da la aplicación de técnicas o prácticas agroecológicas. Esta perspectiva campesina 


\section{Gráfico 1}

\section{Recursos del capital social vinculados a los emprendimientos agroecológicos}

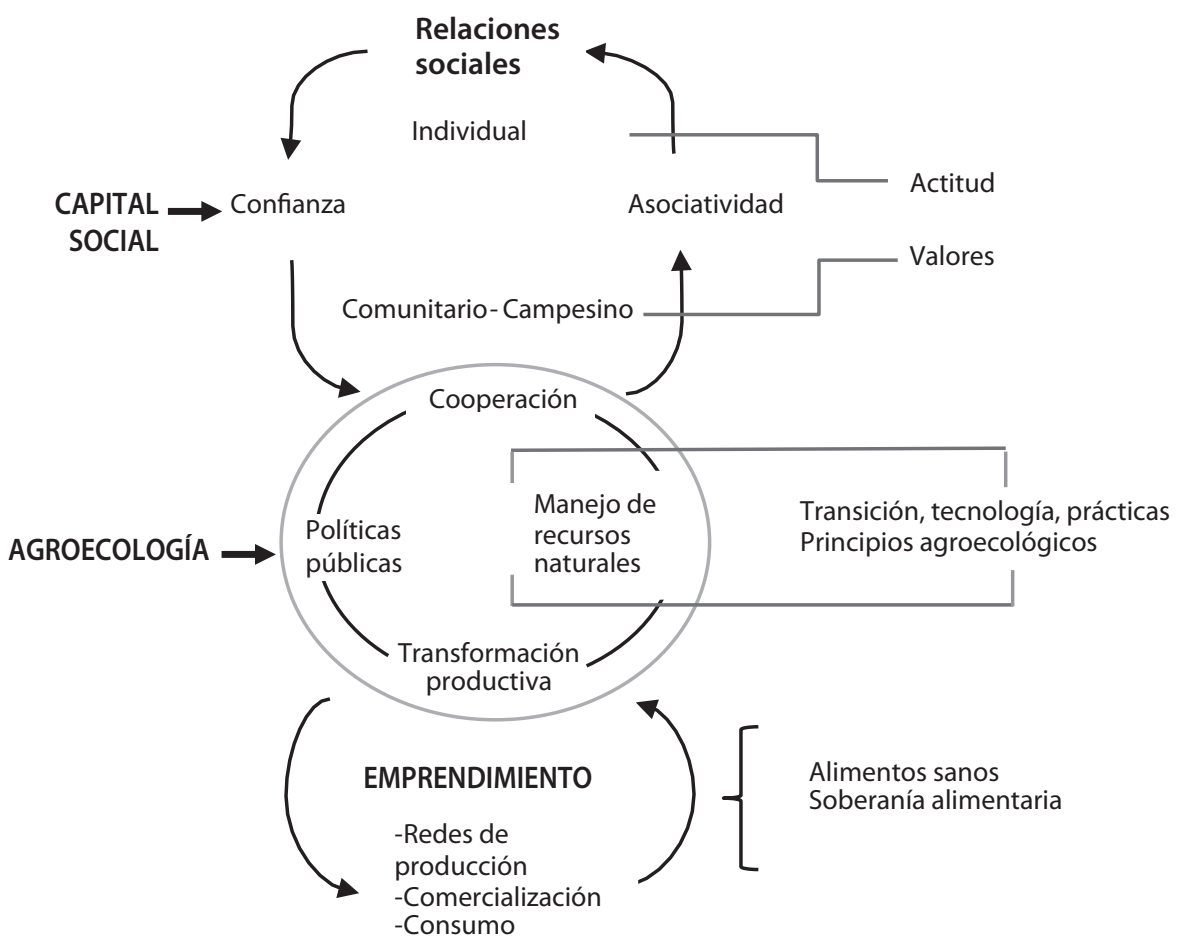

Elaboración propia.

plantea cuatro principios: 1. El sentimiento: parte esencial de la agroecología basado en la conciencia, el amor y el respeto a la tierra, a la naturaleza y a todas las formas de vida; 2. La comunidad: la agroecología recupera y refuerza la confianza y la cooperación dentro y entre las comunidades, grandes o pequeñas, donde supone un cambio de valores que implica pasar del individualismo a la cooperación, defender las relaciones sociales igualitarias y recuperar el sentido comunitario; 3 . Derechos campesinos: derechos como el acceso a las semillas, a la tierra, al agua y a otros comunes son un requisito esencial de la agroecología; y 4. Lucha y transformación social: es necesaria 
la articulación campesina y el fortalecimiento de las bases para avanzar en la propuesta política, en la que la agroecología se constituye en una bandera política de cambio social. Por lo tanto, la agroecología constituye también una propuesta política, asumida por las organizaciones campesinas alrededor del mundo, en su lucha por la soberanía alimentaria.

Desde esta perspectiva, y desde un accionar sociopolítico, Calle y Gallar (2010) señalan que la agroecología incide, en su mirada y en su praxis, sobre los procesos de cooperación que construyen estilos alimentarios (redes de producción, distribución, comercialización y consumo) equitativos y sustentables. Otra óptica del cooperativismo la presenta Kliksberg (2012), cuando, al citar a Graziano Da Silva -director de la FAO para ese momento- (FAO 1994), destaca que este funcionario ve en la potenciación de las cooperativas de agricultores pobres un arma maestra en la lucha contra el hambre. Más adelante refiere que organizaciones como la FAO necesitan cooperativas y organizaciones de productores fuertes como socios clave en el esfuerzo para eliminar el hambre que sufren cerca de mil millones de personas.

Calle, Vara y Cuéllar (2006) plantean un modelo que articula los recursos asociados del capital social comunitario con los emprendimientos agroecológicos. A decir de los autores, es una visión de alcance medio, mas no una omnicomprensiva narrativa social, en torno a tres grandes dimensiones en las que se funden lo ecológico y lo social en aras de dicha transición. Ellas son: a) la micro-socio-cultural o de dinámicas de cooperación, que afecta las dimensiones personales de cambio; b) la sociopolítica o de instituciones, bien sociales o públicas; y c) la ecoestructural o relativa a los circuitos de manejo de recursos naturales y de las tecnologías asociadas que posibilitan dichos circuitos, incluyendo los manejos de unidades productivas.

Cuéllar y Calle (2009) explican que la transformación social agroecológica debería desarrollar relaciones sociales tendientes a obtener una transición agroecológica en los territorios basadas en la equidad, la horizontalidad, la solidaridad y el apoyo mutuo, en lugar de la competitividad y las formas conflictuales que promueve el paradigma económico hegemónico desde su configuración en el liberalismo histórico. Por otro lado, Sandoval (2013) explica que la transformación social, vista desde el ámbito rural, se muestra como un proceso a largo plazo sin resultados finales o acabados y realizados para adaptar a una sociedad a las nuevas dinámicas socioculturales, ele- 
mentos de identidad y pertenencia; es más, se observa que, para alcanzar procesos de transformación productiva, los países desarrollados se enfocan en la investigación y la innovación mientras que los países en desarrollo se concentran en la modificación de su estructura productiva.

Adicionalmente, Lotero y Hernández (2002) plantean que los procesos actuales de transformación productiva suponen la existencia de condiciones favorables que impulsen el empoderamiento y la participación activa de la sociedad hacia dicho objetivo. Por esta razón, se requiere crear condiciones de confianza hacia las instituciones públicas, avanzar del individualismo al colectivismo y promover la eficiencia institucional, entre otros aspectos, que contribuyan a crear un buen clima social y que influyan positivamente en lograr la competitividad sistémica; es decir, incrementando la productividad y garantizando principios de equidad.

En concordancia con estos autores, Rivas (2007) señala que para alcanzar una transformación social agroecológica es necesario tener en cuenta dos condiciones fundamentales. En primer lugar, en el caso del capital social, indica que, si bien la confianza está presente en algunas personas de forma natural o adquirida, y que puede y debe ser trabajada, también esa condición o resultado en acciones cooperativas-solidarias es muy difícil de encontrar o alcanzar.

Por otro lado, señala que para conseguir aportes del capital social como recurso promotor del desarrollo de sistemas de producción agroecológicos se requiere como punto de partida tomar en cuenta los siguientes elementos: a) en los diferentes niveles de gobierno, es necesario elaborar y apoyar políticas públicas de agricultura familiar de cuño agroecológico, visualizando el desarrollo sustentable de parcelas y comunidades agrícolas; b) hacer uso de la voluntad solidaria de las más diversas personas para iniciar trabajos de aproximación, extensión y convivencia que visualicen la sustentabilidad local y la fraternidad universal entre el hombre y la naturaleza; c) aprovechar la experiencia investigativa para trascender el trabajo académico, rescatando valores intrínsecos de solidaridad y respeto para con el prójimo y la naturaleza presentes en la comunidad campesina; d) hacer del trabajo agroecológico y de construcción de capital social una filosofía de vida, unificando práctica y discurso en una sola expresión a través del ejemplo; y e) dar continuidad a las prácticas agroecológicas, transformándolas en un proceso constante de 
recuperación, conservación, supervivencia y cultura que busquen la calidad de vida y la preservación transgeneracional del entorno (Rivas 2007).

Otro aspecto importante en estos procesos es lo relativo al consumo de alimentos como estrategia clave de relación productor-consumidor, a fin de establecer lazos directos a nivel local, permitiendo la conformación de asociaciones o cooperativas autogestionarias y, aunque algunos de ellos tienen un carácter informal, agrupan a personas del mismo territorio con el objetivo de fomentar la relocalización de la alimentación ecológica, mediante las relaciones directas entre consumidores y productores y a través de circuitos cortos de comercialización (Vivas 2010).

De lo anterior se puede deducir que los cambios que se pretendan alcanzar con la transformación productiva implican transformaciones sociales, culturales y ambientales significativas. El estudio del capital social puede hacer un aporte significativo a la construcción de esta visión prospectiva y proactiva de los emprendimientos agroecológicos, basadas en la detección y valoración de las fortalezas y capacidades propias de los grupos, las organizaciones y comunidades campesinas.

\section{Conclusiones}

Las distintas consideraciones expuestas en este trabajo permiten enunciar tres grandes conclusiones. La primera lleva a reconocer que la acumulación de capital social a través de la creación de relaciones de cooperación, confianza y asociatividad en las organizaciones campesinas dedicadas a la producción de alimentos sanos constituye una condición necesaria para hacer viable la gestión de los emprendimientos agroecológicos y una precondición que facilita su inicio; toda vez que las decisiones que toman los agricultores para la transición hacia una producción agroecológica dependen no solo de la tecnología y recursos locales disponibles, sino también de numerosos aspectos del sistema social, económico e institucional circundante.

La segunda conclusión es que, si bien no hay una teoría plenamente consolidada sobre el capital social y solo existen propuestas y sugerencias, no es menos cierto que una cantidad considerable de pensadores coincide en reconocer la importancia y validez que tienen los recursos asociados al capital 
social (cooperación, asociatividad y confianza) en las acciones colectivas de las organizaciones campesinas. Tales recursos son elementos fundamentales para alcanzar una soberanía alimentaria sana a partir de la construcción de estilos alimentarios sustentables.

La tercera conclusión es que, aun cuando existe presencia del capital social en el ámbito rural, se requiere crear condiciones para su despegue; la agroecología no es solo un conjunto de tecnologías conducentes a una transformación de sistemas agrícolas de producción convencional (monocultivos y uso de agroquímicos, entre otras) a sistemas de producción agroecológica (agrobiodiversidad y reciclaje de nutrientes, entre otras), sino también es una trasformación social que implica articular y promover relaciones entre las instituciones y las organizaciones campesinas para el despegue del capital social en procesos de producción, comercialización y consumo. Se debe agregar que no basta con tener capital social sino, ante todo, de cómo se puede potenciar este recurso en un entorno como el medio rural donde los otros capitales como el económico o humano son escasos.

El análisis construido en esta investigación no es en modo alguno exhaustivo, el proceso de conformación teórica es continuo y podrá validarse en medio real; su análisis permitiría llevar acciones encaminadas a expandir los márgenes de oportunidades y bienestar de las organizaciones campesinas, creando un nuevo escenario de desarrollo de los emprendimientos agroecológicos.

Los temas que serían motivo de futuras investigaciones son las siguientes: ¿Cómo determinar el aporte del capital social en transformaciones agroecológicas? ¿Qué indicadores son los que deben medirse en organizaciones campesinas, para conocer su grado de fortalecimiento en actividades no económicas? ¿Qué factores sociales, económicos y políticos inciden en el desarrollo de los sistemas de producción agroecológicos? ¿Cuáles serían las políticas públicas locales que contribuyan al despegue de los activos del capital social en las organizaciones campesinas con emprendimientos agroecológicos? Durante el levantamiento de información en campo de las futuras investigaciones se sugiere considerar los siguientes parámetros como elementos del capital social: grado de confianza asociativa en las organizaciones; grado de involucramiento en toma de decisiones; grado de participación en el cui- 
dado de los bienes comunes; participación en el trabajo de la parcela; y grado de reciprocidad al interior de la organización.

\section{Referencias}

Altieri, Miguel. 1995. "Bases y estrategias agroecológicas para una agricultura sustentable". Agroecología y Desarrollo: Revista de CLADES 8, No. 9: 21-30.

---, y Clara Nicholls. 2007. "Conversión agroecológica de sistemas convencionales de producción: teoría, estrategias y evaluación". Ecosistemas: Revista Científica y Técnica de Ecología y Medio Ambiente 16, No. 1: 12-13.

Ángel, Augusto. 1996. El reto de la vida. Ecosistema y cultura. Una introducción al estudio del medio ambiente. Bogotá: Ecofondo.

Araque, Wilson. 2013. "Factores determinantes de la asociatividad como alternativa para el fortalecimiento de pequeñas y medianas industrias". Tesis doctoral en Administración. Universidad Andina Simón Bolívar, Sede Ecuador.

Arriagada, Irma. 2003. "Capital social: potencialidades y limitaciones: analíticas de un concepto". Estudios Sociológicos: Red de Revistas Científicas de América Latina, El Caribe, España y Portugal 9, No. 63: 557-584.

Berkes, Fikret, y Carl Folke. 1998. Linking Social and Ecological Systems: Management Practices and Social Mechanisms for Building Resilience. Nueva York: Cambridge University Press.

Bourdieu, Pierre. 1985. The Forms of Capital. In Handbook of Theory and Research for the Sociology Education. Nueva York: Cambridge University Press.

Calle, Ángel, y David Gallar. “Agroecología política: transición social y campesinado”. Ponencia presentada en el VIII Congreso Latinoamericano de Sociología Rural ALASRU. Porto de Galinhas, 19 de noviembre de 2010.

Calle, Ángel, Isabel Vara y Mamen Cuéllar. 2006. Soberanía alimentaria: la transición social agroecológica. Barcelona: Icaria.

Caporal, Francisco, y José Costabeber. 2004. Agroecología: algunos conceptos y principios. Barcelona: Icaria.

Carrizosa Umaña, Julio. 2001. ¿Qué es ambientalismo? La visión ambiental compleja. Bogotá: Centro de Estudios de la Realidad Colombiana (CEREC) / Instituto de Estudios Ambientales (IDEA) de la Universidad Nacional de Colombia / Programa de Naciones Unidas para el Medio Ambiente (PNUMA).

Centro de Educación y Tecnología (CET). 2018. Manual de transición para la agricultura familiar campesina. Santiago: IESS.

Coleman, James. 1990. Foundations of Social Theory. Londres: Harvard University Press. 


\section{Marco Polo Cevallos Suarez y José Gregorio Mendoza Mendoza}

Coordinadora Ecuatoriana de Agroecología. 2010. Boletín electrónico. Consulta: mayo de 2018. https://es.slideshare.net/delDespojoCrnicas/boletn-de-la-coordinadora-ecuatoriana-de-agroecologà.

Coordinadora Europea Vía Campesina. 2014. “Agroecología. La transformación social desde la producción de alimentos y la lucha campesina". Consulta: agosto de 2018. 〈http://www. eurovia.org/es/agroecologia-la-transformacion-social-desde-la-produccion-de-alimentos-y-la-lucha-campesina/>.

Crutzen, Paul. 2002. Geology of Mankind. Nature. San Diego: Macmillan.

Cuéllar, Mamen, y Ángel Calle. "Sistemas participativos de garantía: poder, democracia y agroecología". Ponencia presentada en el I Congreso de Sociología de la Alimentación. Gijón, 28 y 29 de mayo de 2009.

Dirven, Martine. 2003. "Entre el ideario y la realidad". En Raúl Atria y otros, comps., Capital social y reducción de la pobreza en América Latina y el Caribe: en busca de un nuevo paradigma, 397-446. Santiago: CEPAL.

Durston, John. 2000. “¿Qué es el capital social comunitario?”. Serie Políticas Sociales, No. 38: $1-44$.

---. 2002. El capital social campesino en la gestión del desarrollo rural. Santiago: ONU / CEPAL.

Farhad, Sherman. "Los sistemas socio-ecológicos: una aproximación conceptual y metodológica". Ponencia presentada en las XII Jornadas de Economía Crítica de la Universidad Pablo de Olavide. Sevilla, 18 de febrero de 2012.

Flora, Jan, y Cornelia Butler. 2003. "Desarrollo comunitario en las zonas rurales de los Andes". En Raúl Atria y otros, comps., Capital social y reducción de la pobreza en América Latina y el Caribe: en busca de un nuevo paradigma, 555-578. Santiago: CEPAL.

Gliessman, Stephen. 2002. Agroecología: procesos ecológicos en agricultura sostenible. Turrialba: Centro Agronómico Tropical de Investigación y Enseñanza (CATIE).

Gómez Limón, José, Esperanza Vera Toscano y Fernando Garrido Fernández. 2012. "Percepción social de los derechos de propiedad agroambiental. El caso de Andalucía". Revista Internacional de Sociología 46, No. 51: 145-175.

Guzmán Casado, Gerardo, Mario González de Molina y Eduardo Sevilla. 2000. Introducción a la agroecología como desarrollo rural sostenible. Madrid: Mundiprensa / Ministerio de Educación y Cultura.

Heifer-Ecuador. 2014. La agroecología está presente. Mapeo de productores agroecológicos $y$ del estado de la agroecología en la sierra y costa ecuatoriana. Quito: Heifer.

Kliksberg, Bernardo. 2002. Hacia una economía con rostro humano. Maracaibo: Universidad del Zulia / Fondo de Cultura Económica.

---. 2012. "La hora de la economía social". Diario (Trujillo), 14 de febrero de 2012, 10. «https://www.pagina12.com.ar/especiales/archivo/bernardo_kliksberg/021-KLIKSBERG. pdf). 
López, Macarena, Fernando Martin y Pedro Romero. 2007. Una revisión del concepto y evaluación del capital social. Madrid: Universidad de la Rioja.

Lotero, Jorge, y Juan Hernández. 2002. "Desarrollo local y regiones rurales en Antioquia: papel de la gestión pública en contextos de transformación productiva": Territorios: Revista Colombiana 7: 109-138.

Martínez, Luciano. 2003. “Capital social y desarrollo rural”. Íconos, No. 16: 73-83.

Miranda, Francisca, y Evelyn Monzó. 2003. Capital social, estrategias individuales y colectivas: el impacto de programas públicos en tres comunidades campesinas de Chile. Santiago: ONU / CEPAL.

Newton, Kenneth. 1997. "Social Capital and Democracy". American Behavioral Scientist: Journal, No. 40: 575-586.

Organización de las Naciones Unidas para la Alimentación y la Agricultura (FAO). 1994. Participación campesina para una agricultura sostenible en países de América Latina. Roma: FAO.

Parker, Cristian. 2001. "Capital social y representaciones socioculturales juveniles: un estilo de jóvenes secundarios chilenos". En Durston y Miranda, comps., Capital social y políticas públicas en Chile, 31-39. Santiago: CEPAL.

Piselli, Fortunata. 2003. "Capital social: un concepto situacional y dinámico". En Arnaldo Bagnasco y otros, edits., El capital social: instrucciones de uso, 538-553. Buenos Aires: Fondo de Cultura Económica.

Pizzorno, Alessandro. 2003. "Por qué pagamos la nafta: por una teoría del capital social”. En Arnaldo Bagnasco y otros, edits., El capital social: instrucciones de uso, 19-52. Buenos Aires: Fondo de Cultura Económica.

Portes, Alejandro. 1998. "Social Capital: Its Origins and Applications in Modern Sociology". Annual Review of Sociology: Journal, No. 24: 1-24.

Programa de la Naciones Unidas (PNUD). 2000. Programa de la Naciones Unidas: informe de desarrollo humano en Chile: asociatividad y capital social. Santiago: PNUD.

Putnam, Robert. 1993. "The Prosperous Community: Social Capital and Public Life". The American Prospect 4, No. 13: 35-42.

Putzel, James. 1997. "Accounting for the Dark Side of Social Capital: Reading Robert Putnam on Democracy”. International Development Journal 9, No. 7: 939-949.

Ramírez, Jorge. 2005. "Tres visiones sobre capital social: Bourdieu, Coleman y Putnam". Acta Republicana Política y Sociedad 4, No. 4: 21-36.

Razzeto, Luis. 2007. “La economía de la solidaridad: concepto, realidad y proyecto”. En José Luis Coraggio, org., La economía social desde la periferia. Contribuciones latinoamericanas, 317-338. Buenos Aires: Altamira.

Rivas, Tirso. 2007. “Agroecología y redes solidarias (capital social): Maracajá, la búsqueda de un desarrollo sustentable en la región semiárida del Nordeste de Brasil”. Tesis doctoral en Recursos Naturales y Gestión Sostenible. Universidad de Córdoba, España. 
Sandoval, Adriana. 2013. "Nuevas ruralidades: expresiones de la transformación social en México". Íconos: Revista de Ciencias Sociales, No. 47: 160-162.

Schejtman, Alexander, y Julio Berdegué. 2004. "Desarrollo territorial rural". Debate y temas rurales: Revista Centro Latinoamericano para el Desarrollo Rural, No. 1: 4-46.

Vivas, Esther. 2010. "Consumo agroecológico: una opción política". Política social: Revista Viento Sur, No. 108: 54-63.

Yurjevic, Andrés. 1996. "El desarrollo sustentable: una mirada actualizada". Agroecología y desarrollo: Revista CLADES, No. 10: 10-17. 